\title{
1/f Noise properties of swift heavy ion irradiated epitaxial thin films of YBCO
}

\author{
S K ARORA*, RAVI KUMAR, D KANJILAL, G K MEHTA, S KHATUA ${ }^{\dagger}$, R PINTO ${ }^{\ddagger}$, \\ VIJAY KUMAR ${ }^{\S}$ and A K GUPTA \\ Nuclear Science Centre, Aruna Asaf Ali Marg, New Delhi 110067 , India \\ ${ }^{\dagger} J a i$ Hind College, Churchgate, Mumbai 400020 , India \\ ¥Tata Institute of Fundamental Research, Homi Bhabha Road, Mumbai 400005 , India \\ ${ }^{\S}$ Superconductivity Division, National Physical Laboratory, New Delhi 110012, India
}

\begin{abstract}
Effect of $250 \mathrm{MeV}{ }^{117} \mathrm{Ag}$ ion irradiation induced columnar defects on the noise properties of the YBCO superconductor in the normal and superconducting state have been investigated. Magnitude of the spectral density of the noise is found to scale inversely with the frequency and exhibit a quadratic dependence on the bias current confirming that the noise arises due to the resistance fluctuations. The magnitude of $S_{\mathrm{y}}$ has been found to decrease with decrease in temperature and shows a noise peak in the transition region. The noise performance of these materials in the vicinity of the superconducting transition as well as in the normal state is found to improve by an order of magnitude after irradiation with $250 \mathrm{MeV}{ }^{107} \mathrm{Ag}$ ions. The decrease in the magnitude of $1 / f$ noise peak is due the irradiation induced enhanced flux pinning of the material which suppresses the flux motion induced noise in the vicinity of $T_{c}$.
\end{abstract}

Keywords. High $T_{\mathrm{c}}$ superconductors; thin films; $1 / f$ noise.

\section{Introduction}

Since from the discovery of high temperature superconductivity (HTSC) there has been a growing interest in the study of conductance fluctuations particularly in the low frequency region ( $1 / f$ noise) (Taesta et al 1988; Maeda et al 1989; Song et al 1990, 1991; Kawasaki et al 1992; Arora and Gupta 1994; Hall and Chen 1994; Bobyl et al 1995; Chen et al 1995). This is due to the reason that strength of conductance fluctuations put a limit to the ultimate sensitivity of the HTSC devices. Moreover, conductance fluctuations often provide information about the scattering process, the structural disorder and phase transitions (Dutta and Horn 1981; Weismann 1988). This is because of the sensitivity of the noise spectrum to the disorder and strain in the structure and phase transitions involved. Strength of the noise technique has been demonstrated previously by changing the amount and structure of disorder by weak annealing or irradiation in metals and semiconductors, yielding orders on magnitude change in noise intensity, whereas the resistivity change was only a few percent (Fleetwood and Giordano 1985; Kirten and Uren 1989; Hooge 1994). In general, HTSCs show large magnitude of noise in the normal state, which is much higher than that observed in the metals and conventional superconductors as predicted by the Hooge's relation (Hooge et al 1981) and the thermal

*Author for correspondence fluctuation model (Voss and Clarke 1976). In the vicinity of the superconducting transition temperature, HTSCs show a sharp rise in the noise magnitude (noise peak) with the decrease in temperature. The anomalous noise peak observed in HTSC is attributed to the large anisotropy and the random flux motion (Arora and Gupta 1994; Chen et al 1995). The noise peak observed in the transition region has tremendous implication on the HTSC devices which operate in the transition region, such as superconducting quantum interference devices (SQUIDs), IR detectors etc (Tinchev 1995; Macfarlane et al 1995). There have been efforts to improve the noise performance of these high- $T_{\mathrm{c}}$ materials and it has been found the sample quality plays an important role in determining the noise magnitude. With the improvement in the sample quality the noise magnitude as well as the amplitude of the noise peak in the transition region decreases (Song et al 1990; Arora and Gupta 1994; Bobyl et al 1995). In order to further improve the noise performance of the HTSC devices one needs to suppress the flux motion induced noise. One way to suppress the flux motion is to introduce appropriate additional pinning centres into the material. This can be efficiently done in HTSC with the swift heavy ions (SHI) irradiation. SHI irradiation of HTSC is known to produce columnar defects (linear tracks) provided the amount of energy deposited by the SHI into the material is above a threshold value. These columnar defects provide strong pinning in the entire range of temperature 
and magnetic field (Civale et al 1991; Budhani et al 1992; Pradhan et al 1996, 1997). Therefore, we have studied the effect of SHI irradiation on the noise properties of $\mathrm{YBa}_{2} \mathrm{Cu}_{3} \mathrm{O}_{7-y},(\mathrm{YBCO})$ thin film microbridges with the motive to understand the role of irradiation induced flux pinning on the noise properties.

In this paper we present a comparative study of the noise properties in the normal and superconducting state of the YBCO thin films before and after irradiation. From the temperature dependence of the noise we observed that the magnitude of the noise in the normal state as well as in the amplitude of the noise peak decreases with the $250 \mathrm{MeV}{ }^{107} \mathrm{Ag}$ ion irradiation. The other noise features remain same such as temperature, frequency and bias current dependence of the spectral density of the noise.

\section{Experimental}

Sample used in the present study was c-axes oriented thin films of YBCO on (100) oriented $\mathrm{LaAlO}_{3}$ substrate. The YBCO thin film was grown in situ using pulsed laser deposition technique, details of which are given elsewhere (Pinto et al 1992). Thickness of the film was $300 \mathrm{~nm}$. The thin films were patterned in the shape of microbridge of width $15 \mu \mathrm{m}$ and length $3 \mathrm{~mm}$ using photolithography and wet etching. Low resistivity contacts were made by depositing silver on the film through the shadow mask. To measure the temperature dependence of the voltage noise and resistivity, standard four-probe detection method was employed. A $\mu$-metal shield was used to screen the sample from the external magnetic field fluctuations. The sample was biased with a battery generated direct current and the voltage developed across it was ac coupled to a low noise preamplifier (SR-552, Stanford, USA). The amplified signal was then applied to a FFT spectrum analyzer (HP35565A, Hewlett Packard, USA) to measure the spectral density of noise and its frequency spectrum. The excess noise arising from the conductance fluctuations in the sample was extracted from the observed noise spectrum by subtracting the background noise (thermal + preamplifier). Details of the cryostat and the experimental set up used for the noise measurements is given elsewhere (Arora and Gupta 1994). To ensure that the contacts do not contribute to the measured noise, we monitored the linearity of the current-voltage characteristics in the normal state during the noise measurements.

Well characterized YBCO thin film samples were irradiated at room temperature with $250 \mathrm{MeV}{ }^{107} \mathrm{Ag}$ ions using 15-DU Tandem accelerator at Nuclear Science Centre, New Delhi (Kanjilal 1997). During irradiation the contact pad area was covered with a thick blob of silver paste to avoid any degradation of the contact. The sample was irradiated with a fluence value $10^{11}$ ions $/ \mathrm{cm}^{2}$.
The irradiation was performed at an angle slightly away from the beam normal to avoid channeling effects. The beam current was kept as low as $0.2 \mathrm{pnA}\left(\sim 1.2 \times 10^{8}\right.$ particles $/ \mathrm{cm}^{2} / \mathrm{sec}$ ) to avoid the heating effects. The ion beam was focused to spot of $1 \mathrm{~mm}$ dia and was scanned over $10 \mathrm{~mm} \times 10 \mathrm{~mm}$ area using a magnetic scanner to irradiate the sample uniformly. To determine the fluence given to the sample, charge falling over the sample surface under the electron suppresser geometry was integrated and the charge pulses were counted with a scalar counter.

\section{Results and discussion}

First of all, it is essential to understand the basic phenomenon of ion transport through a solid target and production of columnar defects (or linear amorphized tracks). It has been well established that when a high energy heavy ion passes through a material, it loses its energy mainly by two ways: electronic excitation (i.e. electronic energy loss $\left.(\mathrm{d} E / \mathrm{d} x)_{\mathrm{e}}\right)$ and direct nuclear collisions (i.e. nuclear energy loss $\left.(\mathrm{d} E / \mathrm{d} x)_{\mathrm{n}}\right)$. In the case of high energy heavy ions nuclear energy loss estimated by Rutherford scattering law is about $2-3$ orders of magnitude less than electronic energy loss due to lower elastic scattering cross-section. Therefore, all the energy loss or energy deposited to the materials can be considered due to electronic excitations, provided the thickness of the material is much less than the stopping range of the heavy ion. Using Standard TRIM95 program (Zeiglar et al 1985) we have calculated the $(\mathrm{d} E / \mathrm{d} x)_{\mathrm{e}},(\mathrm{d} E / \mathrm{d} x)_{\mathrm{n}}$ as a function of energy for ${ }^{107} \mathrm{Ag}$ ion interacting with YBCO as shown in figure 1a. It is clearly evident from figure 1a that $(\mathrm{d} E / \mathrm{d} x)_{\mathrm{c}}$ initially increases with the ion energy and peaks at about $270 \mathrm{MeV}$ in the present case and then decreases with further increase in ion energy. On the other hand nuclear energy loss $(\mathrm{d} E / \mathrm{d} x)_{\mathrm{n}}$ only shows up at lower energies and above $100 \mathrm{MeV}$ it is almost negligible. This implies that the whole energy gets deposited to the thin film in the form of electronic excitations. To achieve the columnar defect we require large $(\mathrm{d} E / \mathrm{d} x)_{\mathrm{e}}$ above a calculated threshold which is $1.2 \mathrm{keV} / \AA$ in the case of YBCO (Szenes 1995). For this purpose, we have selected the ion energy $250 \mathrm{MeV}$ for ${ }^{107} \mathrm{Ag}$ ion beam, which gives $(\mathrm{d} E / \mathrm{d} x)_{\mathrm{e}}$ around $2.5 \mathrm{keV} / \AA$. In figure $1 \mathrm{~b}$ we have plotted the calculated variation of $(\mathrm{d} E / \mathrm{d} x)_{\mathrm{e}}$ for $250 \mathrm{MeV}{ }^{107} \mathrm{Ag}$ ion in $\mathrm{YBa}_{2} \mathrm{Cu}_{3} \mathrm{O}_{7-y}$ target as a function of depth profile. Initially, the variation in electronic energy loss with the depth is very small, i.e. like plateau. Considering the plateau region we have to decide the thickness of the sample. The thin films used in the present study have thickness $3000 \AA$, which is well within the plateau region. Therefore by irradiating the specimens with $250 \mathrm{MeV}{ }^{107} \mathrm{Ag}$ ions we have generated the columnar 
(a)

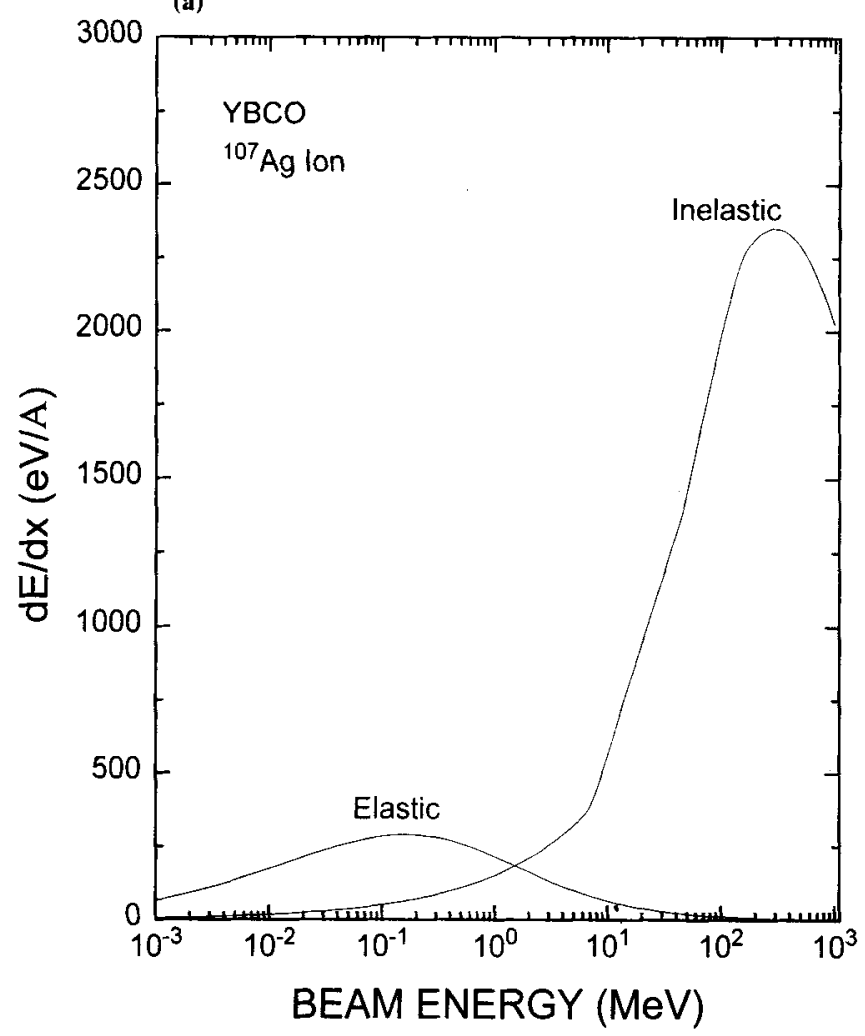

(b)

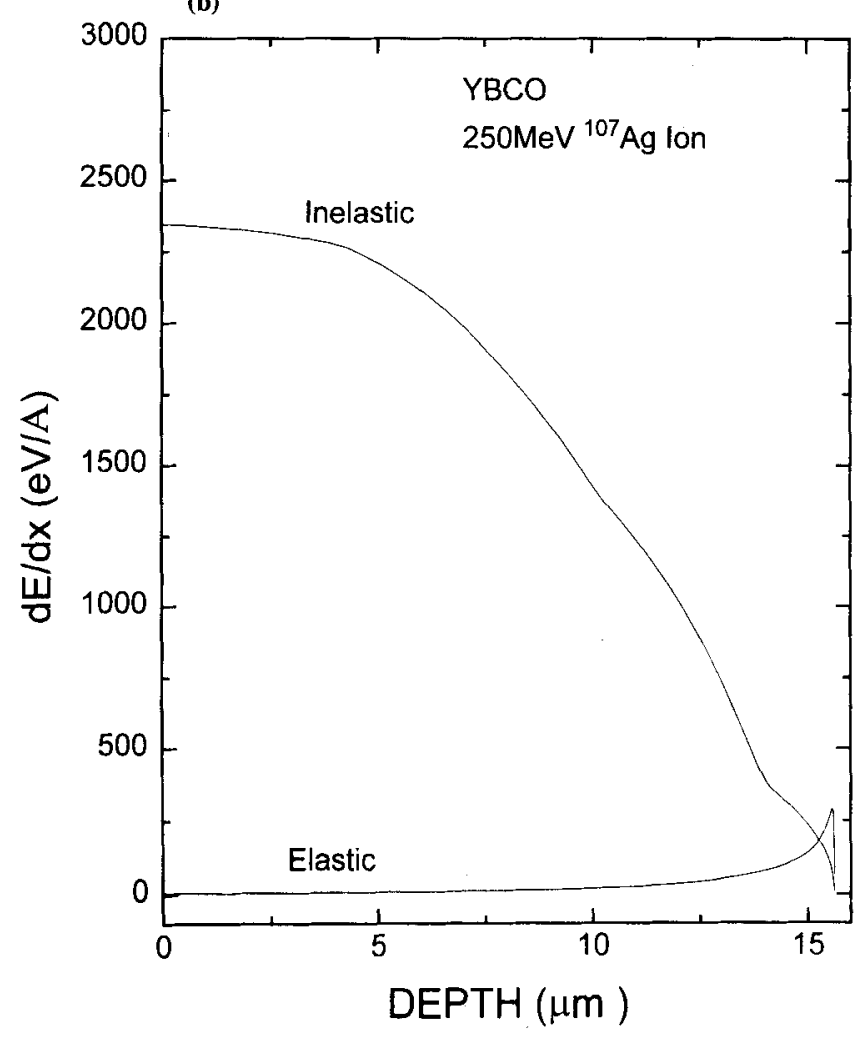

Figure 1. a. Elastic (nuclear) and inclistic (electronic) stopping power as a function of energy for $\mathrm{Ag}$ ion beam in $\mathrm{YBCO}$ and b. elastic and inelastic stopping power as a function of depth for $250 \mathrm{MeV} \mathrm{Ag}$ ions in $\mathrm{YBCO}$. amorphization throughout the whole length of the specimens. The phenomenon of the columnar track production in solids was explained by various workers in the frame work of Coulomb explosion and thermal spike model (Chadderton and Montagu-Pollack 1986; Lesuer and Dunlop 1993). The objective of the present study was to investigate the influence of SHI irradiation induced columnar defects on the noise properties of YBCO high- $T_{\mathrm{c}}$ superconductor.

Electrical resistivity and critical current density of the thin film sample were measured before and after irradiation. Resistivity and $J_{c}$ measurements showed that prior to irradiation the sample had a $T_{\mathrm{c}}$ of $87 \mathrm{~K}$ and critical current density, $J_{\mathrm{c}}$, was $10^{6} \mathrm{~A} / \mathrm{cm}^{2}$ at $77 \mathrm{~K}$. After irradiation with $250 \mathrm{MeV}{ }^{107} \mathrm{Ag}$ ions we observed that the value of resistance in the normal state changed by about $1 \%$ and the $T_{\mathrm{c}}$ of the sample increased by $1.5 \mathrm{~K}$ due to irradiation. Whereas the zero field critical current density was found to decrease by a factor of two.

We have studied the noise properties of several samples before and after irradiation with the $250 \mathrm{MeV}{ }^{107} \mathrm{Ag}$ ion irradiation and present here the results on a sample irradiated with the fluence $10^{11}$ ions $/ \mathrm{cm}^{2}$. This is an optimized fluence for which we have observed the maximum reduction in the noise magnitude in the transition region. Spectral density of voltage noise, $S_{v}$, was studied before and after irradiation as a function of temperature $(77-300 \mathrm{~K})$ in the low frequency region $(0 \cdot 1-50 \mathrm{~Hz})$. Figure 2 shows the voltage noise spectrum of the unirradiated YBCO thin film measured at $300 \mathrm{~K}$. From the figure it is clearly evident that the $S_{\mathrm{v}}(f)$ has $1 / f^{a}$ dependence below $8 \mathrm{~Hz}$ with $\alpha$ value close to 1 . Above this, frequency independent noise (white noise) is observed. Bias current dependence of the $S_{\mathrm{v}}(1 \mathrm{~Hz})$ measured at $300 \mathrm{~K}$ is shown in figure 3. Magnitude of $S_{v}$ is found to have quadratic dependence on the bias

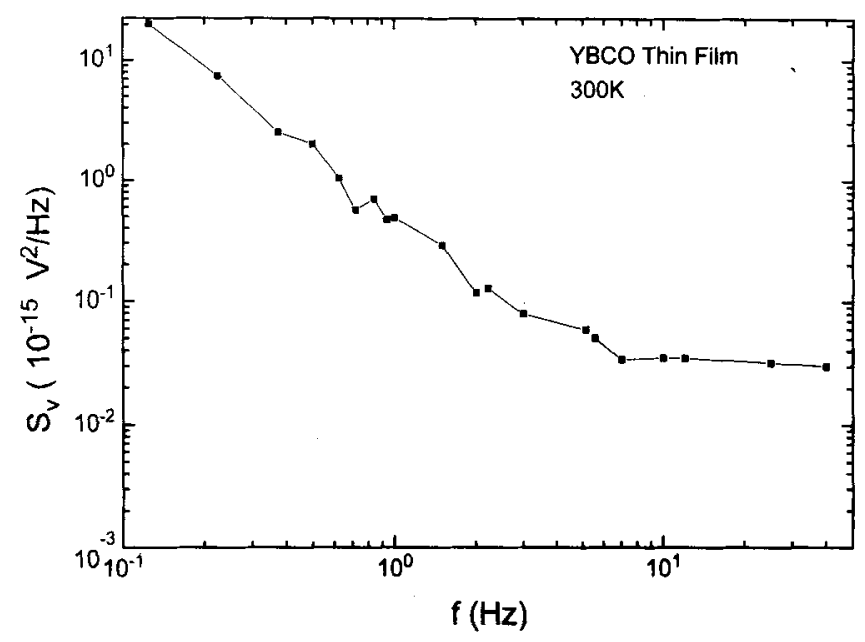

Figure 2. Voltage noise spectra of unirradiated $\mathrm{YBCO}$ thin film measured at $300 \mathrm{~K}$ with a bias current of $1 \mathrm{~mA}$. 
current indicating that the noise arises due to resistance fluctuations. Figure 4 shows the temperature dependence of spectral density of voltage noise, $S_{v}(f)$, in the vicinity of the transition temperature for the YBCO thin film before and after irradiation. It was observed that before irradiation the magnitude of $S_{\mathrm{v}}(f)$ decreased with the decrease in temperature and showed a sharp rise in the noise magnitude in the vicinity of the transition temperature and showed a noise peak. Below this temperature, magnitude of $S_{\mathrm{v}}(f)$ fell below the detection limit $\left(2 \times 10^{-18} \mathrm{~V}^{2} / \mathrm{Hz}\right)$ as the superconducting state was reached. The anomalously large noise magnitude observed in the transition region of HTSC thin films was attributed to the random flux motion and was found to depend strongly on the quality of the material.

Magnitude of the spectral density of voltage noise observed in the normal state of HTSC is much larger than that in metals and low- $T_{\mathrm{c}}$ superconductors. This anomalously large magnitude of the $1 / f$ noise observed in the normal state of high- $T_{\mathrm{c}}$ superconductors is attributed to the large amount of intrinsic defects (oxygen deficient regions, twin planes and stacking faults) and large conduction anisotropy which are inherent to these materials.

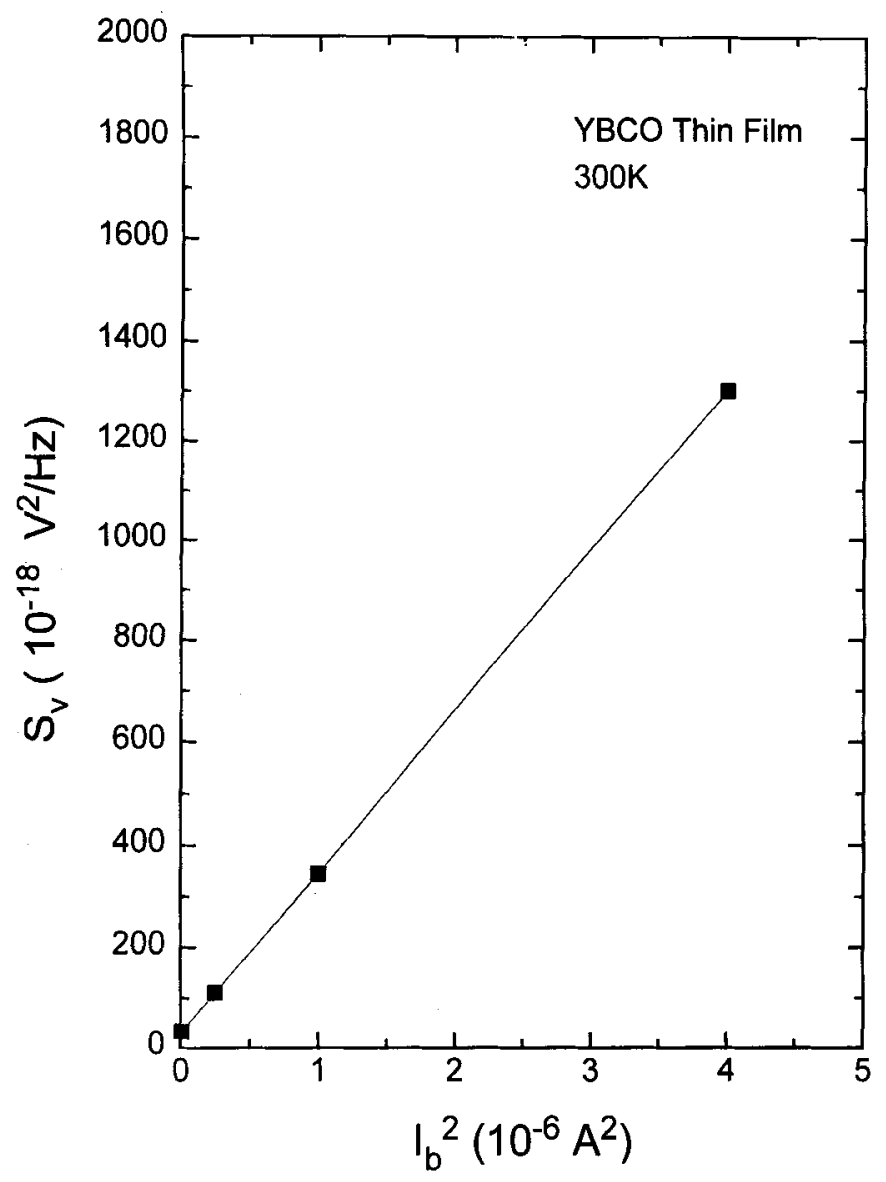

Figure 3. Bias current dependerice of $S_{\mathrm{v}}(1 \mathrm{~Hz})$ for unirradiated YBCO thin film measured at $300 \mathrm{~K}$.
All these factors contribute to the $1 / f$ noise since they lead to the fluctuations in the number density or mobility of the charge carriers. The sample quality also was found to play an important role in determining the extent of the intrinsic noise level and it has been observed previously that with the improved sample quality the noise magnitude decreased significantly. Similar type of results have also been reported by other groups in the YBCO, $\mathrm{Bi}$ and $\mathrm{Tl}$ based cuprate superconducting thin films and single crystals (Song et al 1990; Arora and Gupta 1994; Hall and Chen 1994; Bobyl et al 1995; Chen et al 1995).

In order to compare our noise measurement results with others we have used Hooge's empirical relation for conductance fluctuations (Hooge et al 1981). According to Hooge's relation the spectral density of noise, $S_{\mathrm{v}}$, in a homogeneous material is given as

$$
S_{\mathrm{v}}=\left(\gamma V^{2} / N_{\mathrm{c}} \cdot f^{\alpha}\right)
$$

where $V$ is the dc voltage across the sample, $N_{\mathrm{c}}$ the number of carriers present in the sample, $\alpha$ a constant close to unity and $\gamma$ a dimensionless constant known as Hooge's parameter. The predicted value of $\gamma$ is $2 \times 10^{-3}$, which agrees fairly well with the observed values of $\gamma$ in metals and conventional superconductors (Hooge 1994). We have used a carrier density of $10^{21} / \mathrm{cm}^{3}$ and the experimentally observed value of $S_{\sqrt{ }} V^{2}$. We have found that at room temperature $\gamma$ value for the unirradiated YBCO thin film is 4, which is comparable to the $\gamma$ value reported by other groups (Hall and Chen 1994; Bobyl et al 1995). Figure 4 shows the variation in the magnitude of $S_{\mathrm{v}}(f)$ with temperature of the same film after irradiating with $250 \mathrm{MeV}{ }^{107} \mathrm{Ag}$ ion and was given a fluence of $10^{11}$ ions $/ \mathrm{cm}^{2}$. After irradiation the temperature dependence of the $S_{\mathrm{v}}$ remains similar to that of

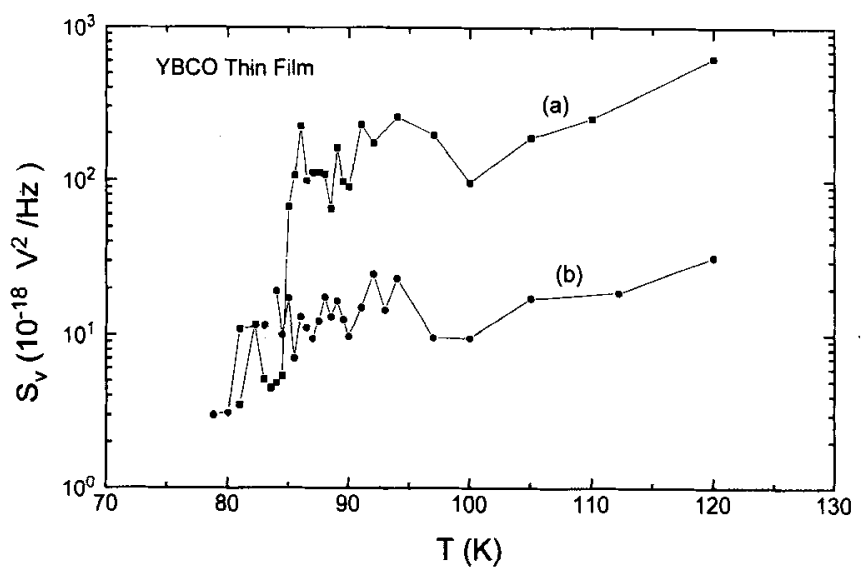

Figure 4. Temperature dependence of $S_{\mathrm{y}}(1 \mathrm{~Hz})$ measured with a bias current of $1 \mathrm{~mA}$ for (a) unirradiated YBCO thin film and (b) after irradiation with $250 \mathrm{MeV} \mathrm{Ag}$ ion with a dose of $1 \times 10^{11}$ ions $/ \mathrm{cm}^{2}$. 
unirradiated specimen. After irradiation with $250 \mathrm{MeV} \mathrm{Ag}$ ions the magnitude of noise in the transition region is found to be one order smaller than that observed prior to irradiation. The value of the Hooge's parameter comes out to be 1 for the YBCO thin film irradiated with $250 \mathrm{MeV} \mathrm{Ag}$ ions at a fluence of $10^{11}$ ions $/ \mathrm{cm}^{2}$. The decrease in $\gamma$ value with the irradiation suggests that the noise performance improves.

The observed modifications in the noise behaviour in the YBCO high- $T_{\mathrm{c}}$ superconducting thin film in the transition region can be understood from the knowledge of the implications of ion transport through the film. As described above, SHI during its passage through a solid target loses its energy via two processes, namely, electronic energy loss, $S_{e}$, and nuclear energy loss, $S_{n}$. If the value of $S_{\mathrm{e}}$ is above a threshold then SHI produces a columnar defect. In the present case the amount of $S_{\mathrm{e}}$ is much above the threshold, therefore, the columnar defects will be produced. These columnar defects act as strong flux pinning centres. Enhancement in pinning efficiency was reported by various groups (Civale et al 1991; Budhani et al 1992; Pradhan et al 1996, 1997). The pinning efficiency of the material is maximum when the size of the columnar defect is of the order of coherence length. In earlier work, it has been shown through STM studies that irradiation of YBCO thin films with $250 \mathrm{MeV}$ produces columnar tracks of diameter about $2 \mathrm{~nm}$ (Ravi Kumar et al 1998). The track diameter is comparable to the coherence length of the material, therefore, one expects that these tracks will provide additional flux pinning centres. Hence the observed noise in the vicinity of the transition region reduces due to the suppression of flux motion which leads to the lowering of the noise. The observed decrease in the noise magnitude in the normal state is possibly due to the irradiation induced release of structural strain.

\section{Summary}

To summarize, we have studied the influence of the 250 $\mathrm{MeV}{ }^{107} \mathrm{Ag}$ ion irradiation on the $1 / f$ noise properties of the YBCO high- $T_{\mathrm{c}}$ superconducting thin film in the normal and superconducting states. We have observed that the magnitude of the noise in the normal state is much higher than that observed for metals and conventional superconductors. The SHI irradiation induced columnar amorphization leads to the lowering of the noise in the transition region due to the suppression of the flux motion induced noise. The improved noise performance in the normal state is possibly due to the release of deposition induced strain via irradiation. We have observed that the value of the Hooge's parameter also reduces with irradiation suggesting that the noise performance of the material is improved.

\section{Acknowledgement}

The authors are thankful to the accelerator group of the Nuclear Science Centre, New Delhi, for their technical support.

\section{References}

Arora S K and Gupta A K 1994 Solid State Commun. 90417 Bobyl A V, Gaevski M E, Khrebtov I A, Konnikov S G, Shantsev D V, Solov'ev V A, Suris R A and Tkachenko A D 1995 Physica C247 7

Budhani R C, Suenaga M and Liou S H 1992 Phys. Rev. Lett. 61985

Chadderton L T and Montagu-Pollock H M 1986 Proc. R. Soc. (London) A274 239

Chen N Y, Jonker R, Matijasevic V C, Jagger H M and Mooji J E 1995 Appl. Phys. Lett. 67133

Civale L et al 1991 Phys. Rev. Lett. 67648

Dutta P and Horn M 1981 Rev. Mod. Phys. 53497

Fleetwood D M and Giordano N 1985 Phys. Rev. B31 1157

Hall $\mathrm{J} \mathrm{J}$ and Chen Tsong-Ming 1994 IEEE Trans. Electron Devices 412128

Hooge F N 1994 IEEE Trans. Electron Devices 411926

Hooge F N, Kleinpenning T G and Vandamme L K J 1981 Rep. Prog. Phys. 4431

Kawasaki M. Chadhuri P and Gupta A 1992 Phys. Rev. Lett. 681065

Kanjilal D, Chopra S, Narayanan M M, Iyer I S, Jha V, Joshi R and Datta S 1993 Nucl. Inst. Methods Phys. Res. A238 97

Kirten M J and Uren M J 1989 Adv. Phys. 38367

Kiss K B and Svedlindh P 1994 IEEE Trans. Electronic Devices 412112

Lesuer D and Dunlop A 1993 Radiat. Eff. \& Defects Solids 126163

Maeda A, Nakayama Y, Takebyashi S and Uchinokura K 1989 Physica C160 443

Macfarlane J C, Hao L, Pegrum C M and Donaldson G B 1995 IEEE Trans. Appl. Supercond. 52212

Pradhan A K, Roy S B, Chaddah P, Kanjilal D, Chen C and Wanklyn B M 1996 Phys. Rev. B53 2269

Pradhan A K, Chen B, Pindoria G, Nakao K, Koshzuka N, Kanjilal D, Chowdhury A J S and Wanklyn B M 1997 Phys. Rev. B55 11129

Pinto R, Pai S P, D'souza C P, Gupta L C, Vijayraghvan R, Kumar D and Sharon M, 1992 Physica C196 264

Ravi Kumar, Samanta S B, Arora S K, Gupta Anurag, Kanjilal D, Pinto R and Narlikar A V 1998 Solid State Commun. 106 805

Song Yi, Misra A, Yue Cao, Querubin A, Chen X D, Crooker P P and Gaines J P 1990 Physica C172 1

Song Yi, Misra A, Crooker P P and Gaines J R 1991 Phys. Rev. Lett. 66825

Szenes G 1995 Phys. Rev. B51 8026

Taesta J A, Song Yi, Chen X D, Globen J, Ik Lee S, Patton B R and Gaines J A 1988 Phys. Rev. B38 2922

Tinchev S S 1995 J. Appl. Phys. 773563

Voss R F and Clarke J 1976 Phys. Rev. B13 4790

Weismann M B 1988 Rev. Mod. Phys. 60537

Zeiglar J F, Bierseck J P and Littmark U 1985 Stopping and ranges of ions in matter (New York: Pergamon Press) 